\title{
Laboreal
}

Volume $7 \mathrm{~N}^{\circ} 2$ | 2011

Varia

\section{Pensar la organización del tiempo de trabajo cuando la tecnología cambia : el caso del equipo de trabajo de operación de una central nuclear}

Pensar a organização do tempo de trabalho quando a tecnologia muda : o caso da equipa operacional de uma central nuclear

Penser l'organisation du temps de travail lorsque la technologie change : le cas de l'équipe de travail d'exploitation d'une centrale nucléaire Consider the organization of the working time when the technology changes : the case study of an operational team in a nuclear power plant

Sophie Prunier-Poulmaire, Aurore Ghesquière, Cecília De La Garza y Charles Gadbois

\section{OpenEdition}

Journals

Edición electrónica

URL: http://journals.openedition.org/laboreal/7518

DOI: $10.4000 /$ laboreal. 7518

ISSN: 1646-5237

Editor

Universidade do Porto

Referencia electrónica

Sophie Prunier-Poulmaire, Aurore Ghesquière, Cecília De La Garza y Charles Gadbois, « Pensar la organización del tiempo de trabajo cuando la tecnología cambia : el caso del equipo de trabajo de operación de una central nuclear », Laboreal [En línea], Volume 7 №2 | 2011, Publicado el 01 diciembre 2011, consultado el 10 octubre 2019. URL : http://journals.openedition.org/laboreal/7518 ; DOI : $10.4000 /$ laboreal.7518

Este documento fue generado automáticamente el 10 octubre 2019

Laboreal está licenciado com uma Licença Creative Commons - Atribuição-NãoComercial 4.0 Internacional. 


\section{Pensar la organización del tiempo de trabajo cuando la tecnología cambia : el caso del equipo de trabajo de operación de una central nuclear}

Pensar a organização do tempo de trabalho quando a tecnologia muda : o caso da equipa operacional de uma central nuclear Penser l'organisation du temps de travail lorsque la technologie change : le cas de l'équipe de travail d'exploitation d'une centrale nucléaire Consider the organization of the working time when the technology changes : the case study of an operational team in a nuclear power plant

Sophie Prunier-Poulmaire, Aurore Ghesquière, Cecília De La Garza y Charles Gadbois

NOTA DEL EDITOR

Manuscrito recibido en : Mayo/2011

Aceptado tras peritaje : Octubre/2011

\section{Introducción}

Este articulo presenta un estudio que aun está en curso en el marco de un proyecto de construcción de una instalación nuclear, el futuro reactor EPR (European Pressurized Reactor) francés. Dicho estudio tiene como objetivo anticipar la organización del trabajo en turnos del personal de operación. La instalación futura está dotada de una 
tecnología avanzada, sin embargo la presencia del hombre $24 \mathrm{~h} / 24$ sigue siendo indispensable para supervisar y operar el proceso. Los horarios por turnos siguen siendo necesarios. Ahora bien, las evoluciones tecnológicas de esta instalación y las transformaciones organizacionales que conlleva para el personal de operación, van a modificar la actividad de trabajo de dicho personal. El futuro operador de la planta se cuestiona sobre el interés de "innovar" en la materia : ¿turnos de 3X8? ¿Turnos de tipo $2 \mathrm{X} 12$ ? ¿O bien una combinación de ambos según el estado del reactor (producción o parada por mantenimiento), o según los días de la semana (fin de semana/semana)?

2 La respuesta a dicha pregunta releva de una problemática general muy bien conocida : la de las actividades de trabajo en horarios por turnos, caracterizadas por su desfasaje en relación con la ritmicidad biológica circadiana, la cual es un dato fundamental del funcionamiento del ser humano, y su desfasaje en relación con los ritmos de la vida social que reglan el desarrollo cotidiano y semanal de las actividades humanas. Las investigaciones llevadas en ese campo muestran que las discordancias tienen incidencias negativas tanto en el trabajo, en términos de fatiga, de eficiencia, de seguridad, como en la vida fuera del trabajo en términos de salud y de calidad de vida personal (Prunier-Poulmaire \& Gadbois 2004, Gadbois, 1998, 2004, Barthe, Queinnec \& Verdier, 2004, Costa, 2010). Estos efectos emergen de manera más o menos importante según las modalidades de los horarios por turnos tales como la hora de principio y final de los turnos en un ciclo de 24 horas, la duración, el ritmo de alternancia entre turnos de día y de noche (Queinnec, Teiger \& de Terssac 1992-2008, Prunier-Poulmaire, Gadbois \& Volkoff, 1998, Barthe, Gadbois, Prunier-Poulmaire \& Queinnec, 2004, Knauth, 2007, Barthe, 2009). Pero, una vez esos datos establecidos, esto no basta para definir un horario de trabajo desfasado lo menos nocivo posible para la salud, ya que como le resume la Figura 1, otros factores intervienen tales como el contenido del trabajo efectuado, las exigencias físicas, mentales y psíquicas (Prunier-Poulmaire \& Gadbois, 2004, Barthe Queinnec \& Verdier 2004, Pavageau, 2006, Barthe, 2009), y las características de la población estudiada : edad, sexo, situación familiar (Gadbois, 2004). Además, esos factores se pueden conjugar y generar efectos variados más o menos importantes; el impacto de uno pudiendo ser modulado por el impacto de otro de manera positiva o negativa. Por ejemplo, el hecho tener hijos a cargo puede disminuir el tiempo de sueño y de descanso diurno, después de una noche de trabajo. Decidir un sistema de horarios desfasados necesita entonces llevar a cabo una reflexión asociando los conocimientos generales sobre el condicionamiento de las actividades laborales por la ritmicidad biológica y la identificación de las exigencias especificas de las actividades laborales consideradas y de las situaciones propias de los trabajadores. Hemos desarrollado aquí un enfoque de ese tipo, basado en el modelo de Queinnec et al. (1992) y completado por Prunier-Poulmaire (1997), para responder de manera prospectiva al cuestionamiento sobre la organización del tiempo de trabajo en el proyecto del futuro reactor francés (Figura 1).

3 Cualquiera que sea el tipo de rotación, éste debe convenir a los diferentes oficios del personal de operación. No se consideran en el estado actual del proyecto ritmos distintos según los oficios por razones de coherencia y de coordinación de las actividades entre los miembros del personal de operación. Sin embargo las características las características del trabajo son diferentes según la función de cada uno. El contenido del trabajo, la carga de trabajo y las fluctuaciones de las actividades que cada uno debe realizar son diferentes. Además, para cada puesto de trabajo las 
características del trabajo varían también según el estado del proceso (en funcionamiento o en paro), los turnos de trabajo, los días de la semana y los momentos del año.

Partiendo de la organización vigente, se trata de prever dentro de lo posible las futuras situaciones de trabajo, sus exigencias y sus riesgos potenciales para contribuir al diseño de turnos satisfactorios. De seguro se necesitaran reajustes ya que el futuro EPR es mucho más automatizado que las centrales nucleares actuales, aparte de que la organización de los equipos de operación es diferente. En las centrales actuales, los oficios del equipo de operación son : el operador reactor, operador vapor, el supervisor, el jefe de explotación y el encargado de las autorizaciones administrativas y un grupo de técnicos. Los futuros equipos de personal de operación serán constituidos de 4 oficios un poco diferentes: el jefe de explotación, un operador acción, un operador estrategia, un delegado de seguridad de explotación (nueva denominación del encargado de las autorizaciones administrativas) y un grupo de técnicos.

Figura 1 - Enfoque multifactorial y sistémico de Quéinnec et al. (1992), completado por PrunierPoulmaire (1997)

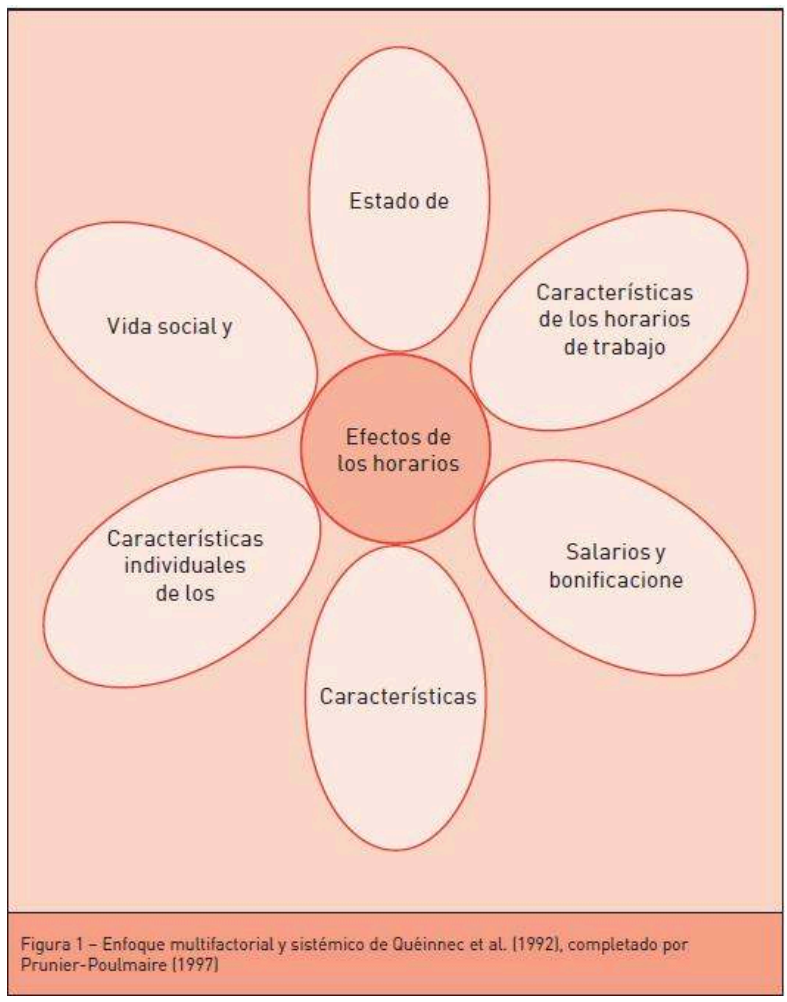

\section{Metodología}

\section{Comprender las características del trabajo de los diferentes oficios del personal de operación para anticipar los mejores horarios por turno rotativos}

Como lo señalan numerosos de autores (Barthe, et al., 2004a, 2004b, Daniellou, 2004 ; Pavageau, 2006; Prunier-Poulmaire, et al. 1998, 2004 ; Quéinnec, et al., 2003), un 
conocimiento preciso y detallado del contenido del trabajo de los operadores que trabajan por turnos es indispensable para poder pronunciar acerca de un tipo particular de horarios por turnos. Según el tipo de actividades realizadas, las consecuencias difieren en términos de vigilancia, fatiga, seguridad, carga y ritmo de trabajo. Las observaciones alimentan la reflexión sobre la organización del tiempo de trabajo aportando elementos concretos que caracterizan la actividad de los diferentes oficios del personal de operación, su medio ambiente de trabajo, las dificultades y riesgos específicos así como el tipo de comunicaciones y coordinaciones necesarias al funcionamiento del equipo de operación, como con los otros servicios y oficios. Este último punto es crucial ya que existen temporalidades distintas entre los servicios de operación y del mantenimiento, ya que este último no funciona por turnos. Esto puede complicar la realización de actividades del trabajo que requieren coordinación y concertación entre los unos y los otros (cf. en este mismo número el articulo de Guillaume Olivier).

6 La instalación nuclear está en construcción, por lo tanto había que encontrar situaciones llamadas "situaciones de referencia" en donde se pudieran realizar un análisis de la actividad del personal de operación. Aunque ni la organización, ni las actividades de trabajo del futuro personal de operación no sean comparables del todo a las actuales, se puede referirse a estas para alimentar la reflexión sobre la organización del tiempo de trabajo. Los resultados obtenidos podrán ser transpuestos a la futura instalación tomando en cuenta los conocimientos que se tienen acerca de las futuras actividades de trabajo previstas. La selección de las situaciones de referencia se hizo privilegiando, entre otras cosas, las plantas más automatizadas, ya que se las considera como las más cercanas a las futuras situaciones de explotación. En total 3 plantas fueron utilizadas como campo de estudio, dentro de las cuales 2 tienen una sala de control computarizada y la última una sala de control convencional. Esto permitió asegurar una cierta coherencia de los datos colectados en las 3 plantas, sobre todo en relación con los oficios de los operadores en la sala de control. Se pudo tener una idea de los elementos considerados como constantes, en cuanto a la actividad de trabajo y su entorno, y de su posible transposición de la situación de referencia a la futura situación que presenta evoluciones tecnológicas mayores. Independientemente del tipo de planta, otros 3 criterios guiaron las observaciones : el estado del proceso, el turno y el oficio.

7 Se decidió observar la actividad de trabajo en periodos de producción y de parada (mantenimiento del reactor) ya que las actividades y carga de trabajo varían de manera significativa. Para cada uno de esos periodos las observaciones se efectuaron en los turnos de mañana, tarde y noche y de manera continua desde la toma de puesto hasta el relevo por el equipo siguiente. Cuando se pudo, se siguió a un equipo durante una semana para cubrir todos los turnos. Los análisis se enfocaron en los 4 oficios que subsistirán en la futura organización :

- El jefe de explotación, responsable operacional de la seguridad de las instalaciones nucleares y un gerente de primer nivel del servicio de operación.

- Los operadores en sala de control, operador reactor y operador vapor, quienes operan y supervisan el proceso nuclear, aunque los roles y misiones de dichos operadores van evolucionar en el EPR.

- El encargado de las autorizaciones administrativas o delegado de seguridad de explotación (nueva denominación), quien es responsable de los actos de explotación que proveen las 
condiciones de seguridad necesarias al personal para poder intervenir en cierto tipo de instalación.

- Les técnicos de terreno quienes aseguran las rondas de supervisión y las maniobras de explotación.

8 En total, el trabajo de campo representa 346 horas de observación de los diferentes oficios. Una parte de la recolección de datos fue común a todos los oficios y otra parte fue especifica. Los puntos que se observaron de manera común son de tipo de manifestaciones de la conducta de baja de la vigilancia (frotarse los ojos, bostezar, estirarse) así como las estrategias para mantener la vigilancia. Se colecto :

- La frecuencia y duración de los intercambios sin relación directa con el trabajo.

- La lectura de documentos sin relación directa con el trabajo en curso.

- El hecho de escuchar música.

- La frecuencia y duración de las pausas durante el turno de trabajo.

- El tiempo de la comida durante el turno.

9 Se relevó también un cierto tipo de indicadores propios de la actividad de los oficios estudiados y se pidió a las personas observadas una serie de explicaciones acerca de su trabajo, cuando esto era posible.

10 En cuanto al Jefe de explotación se centraron las observaciones en las actividades en relación con la seguridad :

- La evaluación e inventario de la seguridad del proceso efectuado en la sala de control.

- El chequeo de los ensayos periódicos de ciertos materiales, que pueden ser semanales, mensuales o solo durante las paradas por mantenimiento.

- El chequeo de los regímenes de las autorizaciones administrativas dando así a los otros servicios el permiso de manera segura de intervenir en ciertos materiales.

- La gestión de los eventos fortuitos (solución de problemas y toma de decisión).

- Las comunicaciones con el Ingeniero de seguridad, quien realiza también una evaluación e inventario de la seguridad del proceso.

11 A lo largo de su turno el Jefe de explotación tiene diversas tareas además de aquellas en relación directa con la seguridad del proceso nuclear : administrativas (participación a reuniones), des gestión del personal (remplazos, cambios en los turnos previstos, ausencias). Dichas actividades se tomaron en cuenta durante las observaciones, así como el hecho de que puede ser solicitado a cualquier momento por el equipo de operación, como por otros servicios durante su turno. Recibe multitud de llamadas telefónicas que se contaron y se midieron en tiempo. Es una persona que se desplaza de manera continua entre la sala de control, la oficina de las autorizaciones administrativas, su propia oficina y puede ir directamente a locales técnicos.

En lo que se refiere a los dos operadores en la sala de control, uno se ocupa del reactor y otro de la parte de las instalaciones relacionadas con los circuitos de agua y vapor. Se realizaron observaciones de ambos centradas en las actividades de operación y supervisión del proceso nuclear. El tipo de actividades de operación que se observo son : ensayos periódicos de instalaciones y material especifico, la baja y la subida de la carga, i.e. las acciones para aumentar o disminuir la producción de electricidad por el reactor en función de la demanda. Las actividades de supervisión del proceso que seguimos son las de la ronda en sala de control (tour de bloc) y la gestión de las alarmas (anticipación y manejo). Se observaron también las actividades de gestión de los eventos fortuitos y se anotaron las comunicaciones telefónicas recibidas y hechas (tipo, 
frecuencia y duración). Los operadores en sala de control computarizada están prácticamente sentados durante todo el turno; en sala de control convencional caminan un poco más, están parados o sentados según las exigencias de la actividad.

El Delegado de la seguridad de explotación realiza las tareas administrativas para preparar las autorizaciones o habilitaciones de seguridad necesarias en cierto tipo de instalaciones. Se centraron las observaciones en el número de habilitaciones de seguridad dadas y recuperadas al final de las intervenciones durante un turno. El número de personas del mantenimiento que vienen a pedir o a dejar una habilitación, la hora a la que pasan a la oficina de las autorizaciones fue anotado, así como las comunicaciones telefónicas recibidas y hechas. El Delegado de seguridad de la explotación se desplaza durante su turno principalmente entre su oficina y la sala de control.

Los técnicos son varios y realizan rondas en las diferentes partes de la central nuclear fuera de la sala de control (la sala de las maquinas, el edificio auxiliar al reactor, etc.). Se observaron las rondas de supervisión y las maniobras de explotación (apertura, cierre des válvulas y otras operaciones de mantenimiento). Se midió también la distancia recorrida durante un turno por un técnico, con un podómetro, ya que se desplazan todo el tiempo para ir de la sala de control a una instalación especifica, luego a otra, etc. Por último se anotaron las comunicaciones telefónicas recibidas y hechas.

El número de personas observadas en total es de 4 jefes de explotación, 20 operadores en sala de control, 6 delegados de la seguridad de explotación y 10 técnicos. De manera paralela la colecta de datos fue completada por 20 entrevistas con personal de operación para confirmar y detallar ciertos aspectos relativos a la dificultad del trabajo por turnos y orientar nuestros análisis de campo según las exigencias propias a cada uno de los oficios.

\section{Comprender las características del trabajo de los oficios del mantenimiento}

16 En el curso de los primeros análisis de la actividad de los diferentes oficios de operación se constató que estos trabajan en estrecha relación con los oficios del mantenimiento quienes funcionan con una organización del tiempo diferente. El personal de mantenimiento trabaja en horarios de día clásicos de 8 h00 AM - 12h00 PM a 1h00 PM 5 h00 PM. Según las actividades previstas, la urgencia de ciertas intervenciones y el estado del reactor (parado o en funcionamiento), el personal de mantenimiento puede estar de guardia y funcionar por turnos de manera puntual.

17 Si bien el servicio de operación y el servicio de mantenimiento son 2 entidades distintas, ambos son fuertemente dependientes uno del otro. Esta diferencia, en términos de organización del tiempo de trabajo, puede causar problemas como retardos en la realización de ciertas actividades. Ante esta constatación, durante el estudio, los futuros responsables de la planta nuclear nos pidieron considerar una mejor conciliación entre las actividades comunes de esos 2 servicios al momento de pensar en el diseño de los horarios por turnos. Nuestras primeras observaciones de campo fueron entonces completadas por una inmersión en el servicio de mantenimiento para identificar las exigencias específicas de dicho servicio en relación con el servicio de operación. El objetivo era poder alimentar la reflexión aportando elementos concretos sobre las obligaciones propias a dicho servicio. Esta inmersión se resume a 
observaciones continuas de las actividades de 2 Encargados de obras eléctricas y de 3 Encargados de obras mecánicas con el fin de identificar el desarrollo de una actividad de mantenimiento en un material y las relaciones con los oficios de operación. Además se realizaron 2 entrevistas con 2 Responsables de equipo (o capataces) para profundizar las cuestiones relativas a los ritmos de trabajo específicos de los oficios del mantenimiento y los lazos con los del servicio de operación.

\section{Anticipar las dificultades a partir de los turnos rotativos vigentes en las 3 plantas}

18 Los turnos rotativos de trabajo de los equipos de operación seguidos son de tipo $3 \times 8$. Se trata de una rotación hacia delante y rápida. Los equipos efectúan siempre 2 o 3 turnos por la mañana, seguidos de 2 o 3 turnos por la tarde y 2 o 3 turnos de noche. El turno de la mañana comienza a las $5 \mathrm{~h} 45 \mathrm{AM}$ y se termina a la $1 \mathrm{~h} 35 \mathrm{PM}$. El puesto del medio día comienza a la 1h15 PM y se termina a las 9h05 PM. El puesto de noche comienza a las 9h45 PM y se termina a las 6 h05 AM. Las rotaciones de los equipos de operación observados se analizaron para comprender la organización prevista de los ciclos de trabajo : duración semanal del trabajo, duración de los turnos, la sucesión de los turnos de mañana, tarde y noche, así como los periodos de descanso y los días efectuados en horarios de día.

19 Aparte de los documentos administrativos que describen la organización de los equipos de operación, se analizaron las rotaciones efectuadas realmente. Esto se hizo con varios objetivos:

- Comparar las rotaciones previstas con las rotaciones realmente efectuadas e identificar desvíos.

- Comprobar que hubo remplazos y tener el número exacto de horas extraordinarias trabajadas más allá de las previsiones iniciales.

- Comprender las razones que indujeron los cambios en las rotaciones e interrogarse sobre los eventuales impactos en los equipos, la actividad de trabajo, la vigilancia, etc.

\section{Conocer las características de la población para anticipar los efectos de los horarios por turno rotativos}

La organización de los horarios de trabajo por turnos rotativos tiene consecuencias en la vida de los asalariados. Esas incidencias no son las mismas según las características individuales del trabajador, de su situación personal y de su vida familiar (Gadbois, 2004). Es por esto que se necesitan conocer las características individuales de la población que trabajara bajo horarios por turno rotativos tales como :

- La edad,

- El sexo,

- La antigüedad en el puesto de trabajo,

- La situación familiar,

- La situación profesional de la pareja,

- El número y edad de los hijos,

- El nivel jerárquico y nivel de sueldo.

21 Dichas características toman sentido en relación también con los datos de la población global de los equipos de operación de las centrales nucleares francesas a nivel nacional. 

(Costa G., 2010). Esto es un punto importante ya que parte de la población contratada para el futuro EPR viene de las otras centrales nucleares. Por lo tanto es necesario ponerse en contacto con el servicio de medicina del trabajo para disponer de un inventario general de la salud de dichos trabajadores. Esto tiene por objetivo identificar si dicha población padece actualmente trastornos descritos bajo la terminología del "síndrome del trabajador en horarios por turno" que abarcan :

- Trastornos del sueño,

- Trastornos cardiovasculares,

- Trastornos gástricos e intestinales,

- Trastornos del humor.

\section{no será tratado en este artículo.}

mismo orden de ideas, los datos relativos a las características personales y a los itinerarios profesionales completos de la población estudiada no se han podido obtener del todo. Así por ejemplo, no se sabe exactamente : ¿cuántas personas del personal de operación ha salido del trabajo por turno en los últimos 10 años y para ir a que puesto de trabajo ? Sin embargo las entrevistas con el personal de operación confirman el carácter exigente y difícil del trabajo por turno rotativo y como esto se acrecienta con la edad, en particular a partir de los 40 años, lo cual confirma los escritos que han destacado este problema desde hace varios años a través la literatura en el ámbito de los horarios por turno (Brugère \& al.1996). El personal de operación señala también la dificultad de poder salir de los horarios por turno sin alternativas interesantes, es decir que no hay una reflexión en relación con los itinerarios profesionales a nivel de los recursos humanos de la empresa. Dicho punto será considerado en nuestro estudio y recomendaciones.

\section{Resultados}

\section{Ritmos de trabajo distintos según los oficios, el estado del reactor, el turno y día de la semana}

Se observa para un mismo oficio un ritmo de trabajo distinto según la hora, el turno, los días y el estado del reactor (en funcionamiento o en paro) y diferencias importantes en términos de contenido y de ritmos de trabajo. Por lo tanto se describirán las características de las actividades que condicionan la carga de trabajo, los ritmos y la duración de las pausas, la vigilancia y las estrategias de los asalariados para mantenerla.

\section{Ritmos de trabajo del Jefe de explotación}

El Jefe de explotación dado su función de responsable de la seguridad en tiempo real, tiene una carga de trabajo elevada cualquiera que sea el turno. Esto se observa a través de numerosas interacciones con las estructuras de operación que se denominan "fuera de turno" y funcionan únicamente de día y el servicio del mantenimiento (reuniones en sala o telefónicas), aparte de análisis de documentos técnicos, y de una exigencia fuerte por parte del personal de operación para tratar puntos técnicos, seguir el trabajo del 
personal en la sala de control y en el resto de la planta. Se le agrega a esto tareas administrativas y de gestión del personal, muchas veces realizadas en el turno de la noche cuando las solicitaciones por parte de la estructura "fuera de turno" y el servicio de mantenimiento ya no están presentes en la planta.

Sin embargo no es tanto el número de actividades que debe realizar el Jefe de explotación que constituye una carga mental en términos de exigencias cognitivas y de estrés, sino sus condiciones de realización. Dado el número de solicitaciones que debe atender, y la necesidad de manejar y resolver los imprevistos que suceden durante los turnos, el Jefe de explotación está involucrado en una gestión permanente de tareas interferentes entre sí, así como en proceso de toma de decisiones importantes relativas a la seguridad de la planta, lo cual puede ser sumamente costoso en términos de exigencias cognitivas.

Para facilitar la toma de decisiones el Jefe de explotación se apoya en parte en los análisis hechos por el Ingeniero de Seguridad, y en parte en los otros oficios del personal de operación, en particular en los 2 operadores de la sala de control para estar al tanto del estado del reactor.

Para terminar, cabe señalar que la totalidad de las tareas inherentes a la función de Jefe de explotación no puede ser realizada durante el turno. Se comprueba un sobrepaso sistemático de los horarios de trabajo de 30 a 6 horas más allá de lo previsto en el turno. A veces dichos sobrepasos de horario se explican por el hecho de tener que participar a reuniones del servicio de operación durante el día.

\section{Ritmos de trabajo de los Operadores Reactor y Vapor}

De manera general, la carga de trabajo y la organización de las actividades de los operadores en sala de control son distintas según el estado del reactor (en funcionamiento o en paro). Cualquiera que se sea el turno observado las actividades de operación que efectúan son múltiples en periodo de paro por mantenimiento, pero esto puede variar según si se está al principio o al final del paro o en fase de puesta en marcha del reactor. Esto se caracteriza entre otras cosas por un número elevado de Ensayos periódicos de ciertos materiales, solicitaciones telefónicas, así como por un manejo de los estados transitorios sensibles que necesitan una atención particular. Sin embargo la concentración que requieren las actividades que realizan no siempre es posible dado el número elevado de personas presentes en la sala de control en periodo de paro por mantenimiento, sobre todo de día. Además, cuando un operador de la sala de control realiza una actividad, es interrumpido frecuentemente por las llamadas telefónicas, 1 en la sala de control por parte del mantenimiento o preguntas de los colegas del mismo servicio y/o equipo de operación. Por lo demás los operadores de la sala de control tienen pocas posibilidades de tomar sus pausas, aparte de la del descanso para comer, en otro ambiente que el de la sala de control, sobre todo en periodo de paro del reactor. Y de todos modos, un operador de la sala de control no puede ausentarse mucho tiempo, ni alejarse de la sala de control por cuestiones de seguridad.

31 Todas las actividades que deben realizar los 2 operadores de la sala de control durante el turno están definidas en un programa. Sin embargo éste no prevé todas las acciones y con frecuencia los operadores tienen que manejar une serie de actividades no previstas. Por ejemplo, la realización de una prueba periódica puede conllevar a una serie de 
problemas técnicos que deben ser resueltos en tiempo real, lo que aumenta el tiempo de realización de dicha tarea y puede implicar otras actividades no previstas tampoco. El programa inicial puede entonces modificarse rápidamente.

\section{Ritmos de trabajo de los técnicos}

32 Una de las características principales de la actividad de los técnicos reside en el hecho de que no hay un turno similar a otro, es decir que no hay una secuencia de las misiones que se repita exactamente entre un turno y otro. Una tal variabilidad en los ritmos de trabajo de los técnicos, cualquiera que sea el turno y el estado del reactor, limita fuertemente la posibilidad de evaluar y anticipar la carga de trabajo durante un turno. Para compensar esta situación, los técnicos desarrollan estrategias en términos de eficiencia, tales como la optimización al momento de realizar las actividades programadas: organizar de manera racional las rondas y maniobras previstas para limitar los desplazamientos y distancias recorridas. Estas estrategias permiten responder más fácilmente a las demandas no programadas (imprevistos) y estar disponible para el resto del equipo de operación, sobre todo en periodo de paro del reactor, cuando el volumen de actividades puede ser más elevado, y no permite tomar descansos, o muy pocos.

Los técnicos tienen una carga física de trabajo substancial pero variable también según las actividades que realizan y el estado del reactor. Dicha carga física se caracteriza por 3 factores : un número significativo de kilómetros recorridos durante el turno (hasta 15 $\mathrm{km}$ ), con transporte de carga pesada en algunos casos, la ejecución de maniobras de explotación necesitando posturas forzadas) (válvulas difíciles de acceso, difíciles de manipular requiriendo fuerza física) y pudiendo estar en zonas radioactivas, lo cual implica precauciones tales como trabajar más rápido para exponerse menos tiempo, tener más cuidado de donde se pone uno para efectuar la intervención, lo cual puede crear otro problema, etc.

A esto se le agrega una carga mental que esta relacionada por una parte con las exigencias cognitivas necesarias para memorizar los diferentes lugares y principales materiales del reactor, y por otra parte con un nivel de concentración necesario al momento de las maniobras para evitar un error.

Cuando el reactor está en marcha parece ser el momento en el cual la actividad de los técnicos fluctúa de manera significativa. Puede haber momentos de baja actividad, sobre de todo en el turno de noche, durante los cuales los técnicos, para mantener la vigilancia, tienen diferentes estrategias (por ejemplo descansar en una salita de control en donde los técnicos ejecutan ciertas acciones).De ese modo pueden reaccionar rápidamente en caso de imprevistos.

\section{Ritmos de trabajo del Delegado de seguridad de explotación}

La oficina de las autorizaciones administrativas resulta ser un lugar estratégico en relación con el desarrollo adecuado de las actividades, tanto para los oficios de operación, como para aquellos del mantenimiento. Los temas de la actividad del Delegado de seguridad de explotación son múltiples : contribuir al interfazaje entre los 2 servicios con temporalidades distintas, y al mismo tiempo garantizar la seguridad de 
las instalaciones. De hecho es uno de los oficios de operación que más interactúa con el servicio de mantenimiento. operación ya que la mayoría de las actividades realizadas por el mantenimiento necesita las habilitaciones de seguridad entregadas por el Delegado de seguridad de la explotación. La organización y ejecución de actividades del mantenimiento dependen de manera precisa de varios puntos.

- La prioridad de la intervención (carácter urgente).

- El personal disponible (variaciones según las vacaciones, la formación, y las ausencias).

- Las aptitudes de cada uno.

- La legislación en vigor (respeto de los tiempos de trabajo/descaso).

los sus actividades, los oficios del mantenimiento están en interacción con los oficios de operación que están de turno. El objetivo de dichas interacciones difiere según el oficio.

Los ritmos de trabajo, distintos entre operación y mantenimiento, tienen como consecuencias situaciones difíciles a la hora de comienzo de los oficios de mantenimiento. En efecto, entre 8h00 AM y 9h00 AM los Encargados de las obras pueden tener que esperar un buen momento (entre 15 y 40 minutos para recuperar los documentos necesarios para sus intervenciones en razón del número elevado de pedidos en la oficina de las autorizaciones administrativas. Entre 1h00 PM y 2 h00 PM cuando los Encargados de la obras van a la oficina de las autorizaciones administrativas 
tienen que esperar que el equipo de operación termine el cambio de turno, el paso de información dado por el equipo que sale y la reunión informativa del equipo que llega, lo cual puede ser largo también.

Además, si las actividades sobrepasan el tiempo del turno, los Encargados de las obras tienen que volver a explicar su intervención al nuevo equipo.

La actividad de los Encargados de las obras se caracteriza por :

- Desplazamientos numerosos que pueden según los días representar una parte importante del tiempo total de la intervención

- Una dependencia con respecto al servicio operación para efectuar una intervención desde el principio hasta el final, es decir desde el momento en que el operador de mantenimiento se presenta en la oficina de las autorizaciones administrativas, recupera la autorización, hasta el momento en que comprueba y prueba el material al final con un técnico del servicio operación.

- Una dependencia con respecto al servicio de operación que puede acarrear tiempos de espera elevado sobre todo en periodo de paro del reactor, que es cuando hay más intervenciones por parte del mantenimiento.

\section{Una gestión de tipo "justo a tiempo" de los turnos rotativos y reemplazos}

Los análisis de la actividad destacan diferencias importantes entre los turnos rotativos previstos y los turnos hechos por el servicio operación.

Si estos desvíos se explican en parte por una serie de remplazos de colegas que se van de vacaciones, por ejemplo durante el periodo estival, cabe señalar que no es la única causa. En ciertas plantas hay una falta de personal, lo cual implica una solicitación del personal de operación durante todo el año y no solo en el periodo estival para completar los equipos. Otros aspectos entran en línea de cuenta ya que los remplazos y las horas extraordinarias significan ventajas financieras.

Sin embargo, la gestión de tipo "justo a tiempo" de los remplazos puede tener consecuencias directas en dos niveles:

- En la producción, ya que los equipos, ante cierto tipo de actividades o no los hacen o les toma más tiempo dada la recomposición del equipo (complementariedad de los conocimientos y experiencias en un equipo en el cual todos se conocen bien, necesarias para poder realizar la actividad en mejores condiciones).

- En la salud ya que a veces el tiempo de descanso es demasiado corto entre los turnos. El sueño puede alterarse tanto desde el punto de vista cuantitativo como cualitativo. A largo plazo, esto puede tener un efecto en la fatiga y en el nivel de vigilancia y por lo tanto en la actividad global de los operadores.

- Aparte, de manera indirecta, la gestión de tipo "justo a tiempo" de los remplazos y la práctica de turnos rotativos que cambian todo el tiempo en relación con lo previsto pueden tener un efecto en la seguridad (error posible, omisión de una acción...). Aunque no se pueden establecer lazos de tipo "causa-efecto" entre el nivel de vigilancia, la salud, la fatiga y la seguridad, ese riesgo no se puede ignorar.

Los turnos rotativos son entonces un instrumento de regulación individual (regulación de las vacaciones, regulación salarial) sobre todo para 3 de los oficios de operación 
(operadores de la sala de control, técnicos y Delegado de seguridad de explotación). Este punto tiene que ser integrado en la reflexión global sobre el tiempo de trabajo.

\section{Aplicación efectiva del modelo de enfoque multifactorial y sistémica : evolución del modelo y falta de algunos datos}

Con respecto de los resultados obtenidos, he aquí cómo evoluciona el modelo del enfoque multifactorial y sistémico (Quéinnec et al., 1992; Prunier-Poulmaire et Gadbois, 1997) (Cf. Figura 2).

Figura 2 - Evolución del modelo del enfoque multifactorial y sistémico (Quéinnec et al., 1992 ; Prunier-Poulmaire et Gadbois, 1997) a raíz de su aplicación

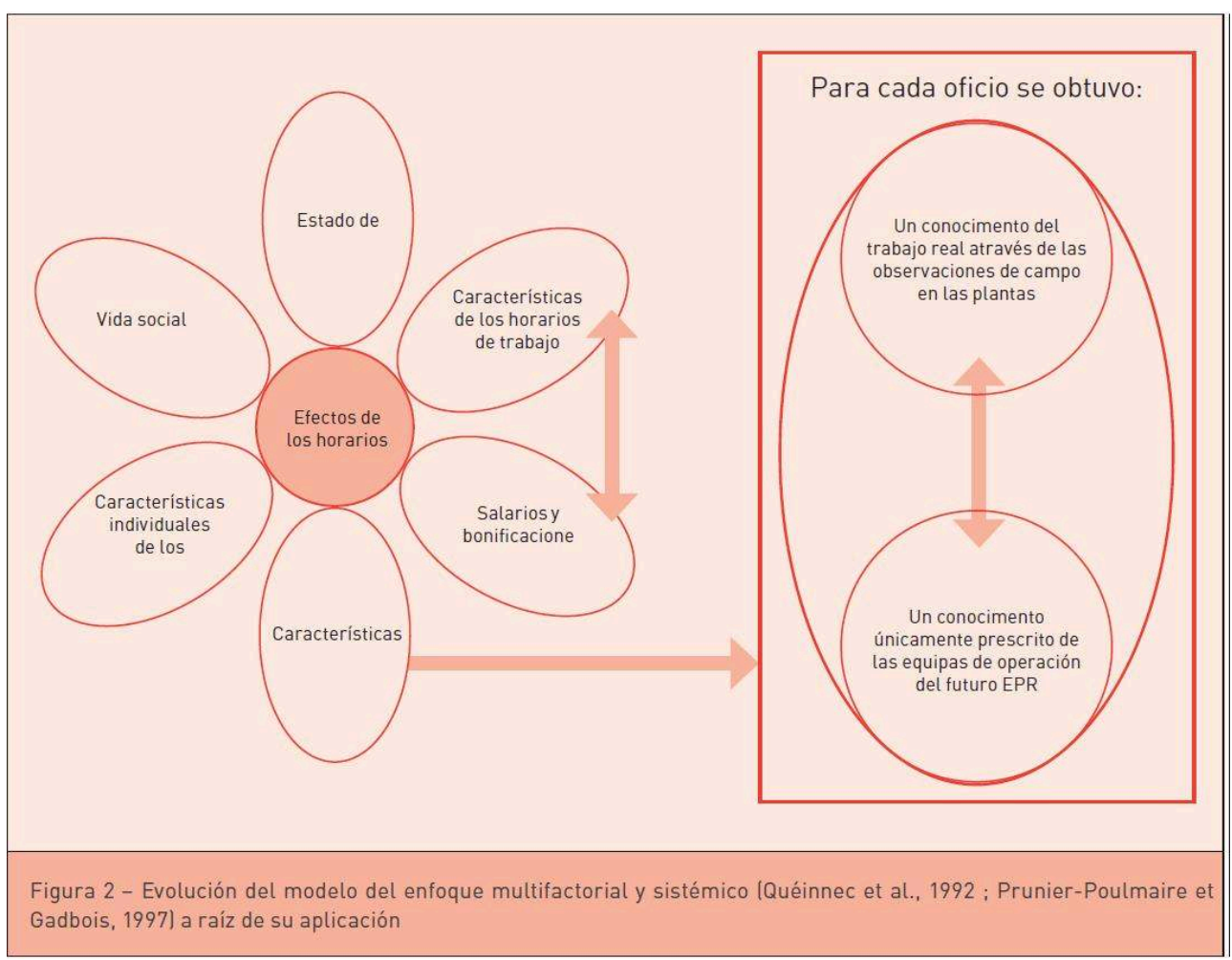

51 Los elementos relativos a las características individuales de los asalariados, la vida social y de familia y la salud se ven voluntariamente reducidos en esta modelización para mostrar que aun no disponemos de la totalidad de dichos elementos.

Se introdujo une flecha entre las características de los horarios de trabajo y el sueldo y bonificaciones para insistir en el vínculo importante que existe entre esos dos elementos. Como se había mencionado anteriormente, la práctica de un turno rotativo con remplazos numerosos y horas extraordinarias aumenta de manera significativa el ingreso del personal de operación (salvo el Jefe de explotación).

Para terminar, en las características del trabajo se introdujo una distinción. Por una parte los conocimientos de las características del trabajo "real" de los oficios de operación obtenidos a través las observaciones de campo en las 3 plantas. Por otra parte, los conocimientos bastante "teóricos" por el momento sobre los roles prescritos de cada uno de los oficios previstos en los futuros equipos de operación del EPR. Estos últimos tendrán que ser alimentados y comparados con los anteriores para tener una 
idea más precisa de los que puede ser o no transferido y utilizado en la reflexión sobre la organización del tiempo de trabajo.

54 La aplicación del modelo y los resultados que se derivan muestran la complejidad que caracteriza el campo de la organización del tiempo de trabajo y las dependencias fuertes entre las exigencias y características del trabajo, los recursos humanos, la salud y características de la población, así como la vida social y de familia.

55 Siete puntos clave tienen que ser considerados en la reflexión sobre el tema de la organización del tiempo de trabajo.

1. La necesidad de identificar el tipo de vínculos entre la duración de los turnos, la carga y ritmo de trabajo y la vigilancia. Se trata aquí de tomar en cuenta los efectos de la duración de los turnos, según el oficio, en la carga mental y/o física del trabajo, pero también en la posibilidad de mantener un estado de vigilancia adecuado.

2. La necesidad de iniciar une reflexión especifica por oficio de operación, más que una reflexión global, dadas las exigencias propias a cada oficio y los ritmos de trabajo propios también. Se trata aquí de encontrar, en un mismo turno rotativo, el mejor compromiso posible para cada uno de los oficios de operación. El caso del Jefe de explotación es interesante para ilustrar este punto ya que es el único que sobrepasa de manera sistemática los horarios de trabajo previstos. Y a pesar de que esto se tolere en las plantas, se puede uno cuestionar sobre los efectos a largo plazo, en términos de fatiga, los efectos sobre la vigilancia y la toma de decisión en sistema de alto riesgo, y dadas sus responsabilidades.

3. El tema del tipo de turno en relación con los problemas de personal o mejor dicho de falta de personal, y el manejo de las ausencias, vacaciones y remplazos. Se debe de analizar de manera global los efectos de la gestión de los recursos humanos en todo turno de trabajo, cualquiera que sea éste $(3 \times 8$ o $2 \times 12)$. Recordamos que en ninguna de las 3 plantas se observaron turnos rotativos que correspondieran a los previstos, cualquiera que fuese el periodo del año. Estos cambios intervienen sin que los efectos en el trabajo, ni menos en la salud y la vigilancia, sean realmente evaluados. Según las entrevistas realizadas, esta situación parece ser general en todas las plantas en Francia.

4. Los efectos de la duración de los turnos, la carga de trabajo al momento de cambiar de turno y la calidad de las informaciones transmitidas al equipo que toma el puesto. Este punto está relacionado con una reflexión sobre los vínculos entre el tipo de turno y los cambios de turno. Por ejemplo si un turno de tipo 2x12 aumenta la duración del turno, pero reduce el número de cambios de turno, es necesario asegurarse que esto no perjudica la calidad de los cambios de turno que tendrán que ser un poco más largos. No hay que olvidar que la calidad de las informaciones transmitidas en los cambios de turno de un equipo a otro, dependen también de la fatiga, y que la fatiga resulta de la interacción compleja entre la duración del turno y de la intensidad de la carga de trabajo de éste.

5. Los efectos de un periodo de descanso - o de un periodo fuera de turno más o menos largo sobre la apropiación del estado del reactor. Este aspecto debe ser considerado en una reflexión sobre la manera más adecuada de alternar entre trabajo/descanso/formación profesional continua y esto desde la fase más temprana del diseño de los futuros turnos rotativos. Si las rotaciones rápidas son hoy en día consideradas como preferibles desde el punto de vista cronobiológico, es por el costo potencial que representa para el organismo la alternancia de periodos largos de trabajo y de descanso. Los esfuerzos requeridos en términos de adaptación y luego de desadaptación progresiva a los ritmos lentos son conocidos como costosos para el organismo. Se puede entonces suponer que periodos largos de exclusión del ritmo de trabajo habitual (para la formación por ejemplo) provoquen trastornos al organismo, dadas las exigencias en términos de esfuerzos de re-sincronización posterior. 
6. El tema de los ritmos de trabajo y de la co-actividad entre el servicio de operación y el de mantenimiento ya sea cuando el reactor está en funcionamiento o en paro. La co-actividad se integra en la reflexión sobre la organización llamada "fuera de turno" en relación con los turnos rotativos y otras obligaciones y exigencias del trabajo. Esta exige involucrar una reflexión de manera simultánea y global sobre la conciliación eficiente de los horarios de trabajo de los equipos de operación y los del mantenimiento, que favorezcan un funcionamiento cooperativo óptimo.

7. El tema del ritmo de trabajo y del tipo de turno rotativo al momento de la puesta en marcha del reactor y en los primeros momentos de producción. La carga y ritmos de trabajo serán seguramente elevados e intensos al momento de la puesta en marcha del reactor, al mismo tiempo que los equipos de operación tendrán que familiarizarse con el nuevo proceso, desarrollar nuevas aptitudes y estrategias para operar, supervisar, etc. El tipo de turno seleccionado puede tener un papel muy importante en ese contexto específico. Se deberá tener cuidado con ese periodo de transición de la actividad.

Estos 7 puntos sintetizan las principales esferas que tendrán que ser objeto de hincapié en la reflexión sobre el tiempo de trabajo. Desde luego que no son exhaustivas y que tienen que ser profundizadas y desarrolladas posteriormente en grupos de trabajo antes de la puesta en servicio de la planta.

\section{Claves para la definición de turnos rotativos de trabajo}

En la fase actual del diseño del proyecto el futuro operador de la planta aun no ha tomado una decisión definitiva en lo que se refiere a la composición de los equipos. El objetivo de este estudio era él de elaborar un marco con el fin de guiar el diseño de los horarios por turno de los futuros equipos de operación. Este marco consta de una serie de claves presentadas y discutidas brevemente en esta parte. Dichas claves serán integradas en los grupos de trabajo previstos sobre la organización del tiempo de trabajo para alimentar la reflexión con los ejecutivos, representantes de la dirección y de los futuros equipos de operación y tal vez hasta del mantenimiento.

\section{Claves teóricas de conocimientos sobre los horarios por turno}

Los claves que proveemos son propuestas en base a los conocimientos científicos provenientes de la literatura nacional e internacional. Se trata de recordar las "reglas fundamentales" que presiden el diseño de un turno rotativo, con el fin de limitar la desincronización de los ritmos biológicos de los asalariados, y de disminuir los efectos sobre su salud, la vida social y de familia.

\section{Claves sobre el conocimiento de cada uno de los oficios del equipo de operación}

59 Estas claves son el resultado de los análisis de campo llevados a cabo en las 3 plantas de referencia, y que fueron confrontados a posteriori con las personas observadas para validarlos.

60 Nuestro propósito se ilustra aquí con algunos de los resultados en los que se debe hacer hincapié en el ámbito de la reflexión sobre los horarios por turno rotativos, tomando en cuenta cada uno de los oficios del equipo de operación. 
61 Desde el punto de vista de los ritmos de trabajo en el curso de los diferentes turnos, se observaron situaciones muy distintas según los oficios. Cabe señalar que los Jefes de explotación hacen más de 8 horas de turno y no toman a veces ni 10 minutos de descanso durante el turno, nunca se le vio tomar el tiempo de comer en ninguno de los turnos observados. Los operadores de la sala de control pueden tener turnos muy cargados durante los cuales es difícil o imposible tomar un descanso. El tiempo para comer es máximo de 30 minutos en un turno "tranquilo" pero puede ser de 10 minutos en un turno cargado. Los momentos de descanso son posibles más fácilmente porque son 2 en la sala de control, y hasta 3 en periodo de paro del reactor.

En cuanto a los técnicos, es prácticamente imposible anticipar la carga de trabajo, aunque esta es más elevada en periodo de paro del reactor. Además su actividad incluye una carga física que tiene un efecto suplementario en la fatiga en ciertos turnos. Los momentos de descanso son viables, pero variables también según el estado del reactor y los lugares de la planta en donde se encuentren. Cuando están por ejemplo en un lugar cuyo acceso es difícil o complicado (como en la zona reactiva que necesita el paso por un vestuario, cambiarse de ropa, etc.), y si saben que tienen que seguir trabajando allí, prefieren no salir y esperarse a poder tomar un momento de descanso más tarde.

En lo que se refiere al Delegado de seguridad de explotación su actividad se diferencia claramente entre los turnos de noche y de los de día. La noche se caracteriza por una actividad más bien administrativa, y el día por una gestión des autorizaciones administrativas con los oficios del mantenimiento. La carga de trabajo más elevada en periodo de paro del reactor, como los otros oficios. Sin embargo este personal puede arreglárselas para tomar un momento de descanso ya que son dos en la oficina en el periodo de paro.

Estos elementos propios a cada uno de los oficios de operación estudiados podrán contribuir a la elección de las opciones las más adecuadas en términos de horarios por turno rotativos y por ejemplo entre un horario de tipo $3 \times 8$ y un horario de tipo $2 \times 12$. Si para algunos de los oficios la segunda opción parece ser viable, para otros como los técnicos, tiene que ser discutida. De hecho este tema tiene que ser analizado de manera precisa en todos los casos y tomando el cuenta la evolución de la organización del equipo de operación prevista.

\section{Claves con relación a las características de la futura población}

Hemos insistido aquí en el interés de un análisis de las características de la población y del seguimiento médico de las personas que trabajan en horarios por turno.

En efecto se sabe que con la edad, los asalariados en horarios por turno soportan cada vez menos los ritmos de trabajo atípicos, sobre todo si la antigüedad en los horarios por turno es elevada (Brugère et al. 1996). Se necesita entonces anticipar el desarrollo de una carrera profesional, de un itinerario profesional que prevea la posibilidad de poder salir de los horarios por turno, proponiéndoles puestos de trabajo en los cuales dicha población pueda continuar a desarrollar sus aptitudes y valorizar su experiencia.

El análisis de las características de la población y su seguimiento médico tienen que realizarse de manera regular para seguir de cerca la evolución de la población, identificar los casos que presentan problemas y ser capaz de anticipar la gestión de las carreras profesionales y aptitudes y experiencia de cada uno de las personas. 


\section{Claves organizacionales y de management}

El tema principal de dichas claves organizacional y de management es el de argumentar la necesidad de optar une gestión rigurosa de los turnos y de los remplazos. En efecto durante nuestras observaciones de campo en las 3 plantas se constataron desvíos importantes entre el turno previsto y él que realizaban los equipos. Y al analizar los turnos del año se comprobó que eso no era puntual sino que formaba parte de la rutina. Como se señalo anteriormente, la práctica de turnos que cambian todo el tiempo, sin tomar en cuenta el funcionamiento crono-biológico, puede tener consecuencias mayores tanto en la salud de los asalariados, como en la fiabilidad del sistema de trabajo.

\section{En conclusión}

69 El estudio presentado aquí ilustra los aportes de la ergonomía a la gestión de los riesgos en el diseño en este tipo de proyecto. El primer punto fuerte de esta gestión se apoya en la anticipación de los riesgos en relación con los horarios por turno rotativos en el plano de la salud y de la seguridad, basándose en los conocimientos relativos a las variaciones circadianas del funcionamiento del ser humano. Esto es el caso por ejemplo de ciertos parámetros de los horarios por turno como las horas de principio y de fin del turno de noche y de la mañana y el ritmo de alternancia.

70 Pero esta anticipación no puede limitarse a una simple transposición directa ya que dada la generalidad de las claves fundadas en los análisis cronobiológicos, estas no consideran las características singulares propias a cada contexto profesional, el cual tiene sus propias exigencias físicas y mentales, en relación con las tareas asignadas a los asalariados y a sus variaciones temporales a la escala de un ciclo diario. Para que la anticipación de los riesgos relacionados con un tipo de organización del tiempo de trabajo sea pertinente se tienen entonces que articular los fenómenos cronobiológicos generales y las características específicas de las actividades realizadas por los operadores. Y este es el aporte indispensable del análisis ergonómico de las actividades de los operadores en las situaciones de referencia, segundo punto fuerte de la gestión de los riesgos.

71 El segundo aporte de este análisis de las actividades en las situaciones de referencia reside en la identificación de elementos de riesgo relacionados con la organización del tiempo del trabajo, no tanto poniendo a prueba la ritmicidad circadiana del ser humano, sino a través de la dinámica del conjunto de tareas de las diferentes categorías de personal. La distribución temporal de las tareas no es la misma de un oficio a otro y, para cada uno de ellos dichas tareas presentan variaciones significativas a la vez en la escala de las 24 horas y en la de las fases del funcionamiento del reactor. El funcionamiento de las plantas implica interacciones entre operadores que tienen que colaborar cuando las actividades propias de cada uno están sometidas a exigencias temporales distintas. Es el caso por ejemplo de las articulaciones entre las actividades de los operadores en la sale de control y los técnicos.

72 Para terminar otro problema identificado a través de los análisis de campo es el desvío casi permanente que existe entre los turnos previstos y los turnos efectuados, lo que puede generar situaciones de funcionamiento no óptimas. 
73 En este punto del estudio se ha logrado constituir una solida base de información conjugando a la vez los conocimientos generales sobre los horarios en turno rotativos y la identificación de elementos de riesgo a través las investigaciones de campo en las situaciones de referencia. A partir de ese material se podrá, mediante los grupos de trabajo previstos por la empresa, instruir y definir diferentes hipótesis en cuanto a turnos posibles, que presenten los mejores compromisos para los asalariados de la futura planta. Sin embargo, cabe señalar por una parte, que nuestro estudio no pudo ir hasta donde queríamos ya que faltan informaciones primordiales tales como la estructura precisa de los equipos. Esto puede limitar de cierta manera el aporte de las claves. Por otra parte la identificación de las situaciones de referencia, indispensables en nuestro enfoque, es un ejercicio relativamente complejo ya que las informaciones colectadas sobre la planta en construcción son parciales y en evolución. La transposición de los resultados de este estudio a las situaciones futuras de operación del EPR va a necesitar seguramente otro tipo de análisis complementario sobre la organización específica de los equipos de operación del EPR.

La participación de los ergónomos en este estado de un tal proyecto conlleva a partir de ahora y en adelante a asociar a los representantes de los recursos humanos, a la medicina del trabajo y de manera más global al staff de dirección a la reflexión sobre el tema de la organización del tiempo de trabajo, considerando la inseparabilidad del contenido del trabajo y de la dinámica temporal en la cual se desarrolla. Esto incita a un trabajo común para una gestión efectiva de la salud y de la seguridad en el trabajo, asociando una anticipación de los itinerarios profesionales de los futuros equipos de operación.

\section{BIBLIOGRAFÍA}

Barthe, B., Gadbois, C., Prunier-Poulmaire, S. \& Quéinnec, Y. (2004). Travailler en horaires atypiques. In Falzon, P, Ergonomie, (pp. 129-144). Paris : PUF.

Barthe, B. (2009). Les $2 \times 12 \mathrm{~h}$ : une solution aux conflits de temporalités du travail posté ? http// Temporalités.revues.org/idex 1137.html

Barthe, B., Quéinnec Y., \& Verdier, F. (2004). L'analyse de l'activité de travail en postes de nuit : bilan de 25 ans de recherches et perspectives, Le Travail Humain, 67 (1), 41-61.

Béguin, P. (2004). L'ergonome acteur de la conception In P. Falzon, (pp. 375-390), Ergonomie. Paris : PUF.

Costa, G. (2010). Shiftwork and health : current problems and preventive actions. Safety and health work, 112-123.

Daniellou, F. (2004). L'ergonomie dans la conduite de projets de conception de systèmes de travail. In P., Falzon. Ergonomie, (pp. 359-374). Paris : PUF.

Gadbois, C. (1998). Horaires postés et santé, Encyclopédie Médico-chirurgicale-ToxicologiePathologie Professionnelle, (16-785-A10). Paris : Elsevier. 
Gadbois, C. (2004). Les discordances psychosociales des horaires postés : questions en suspens. Le Travail Humain 67 (1), 63-85.

Knauth, P. (2007). Extended work periods, Industrial Health, 45, 125-136.

Pavageau, P. (2006). Les effets conjoints du travail et des horaires alternants sur la santé des agents de surveillance des établissements pénitentiaires. PISTES, 8 (2), 1-30. http:// www.pistes.uqam.ca/v8n2/articles/v8n2a3.htm

Prunier-Poulmaire, S. (1997). Contraintes des horaires et exigences des tâches : la double détermination des effets du travail posté. Santé et vie socio-familiale des agents des Douanes. Thèse de Doctorat, Laboratoire d'ergonomie EPHE, Paris.

Prunier-Poulmaire, S., Gadbois, C., \& Volkoff, S. (1998). Combined Effects of shift systems and work requirements on customs officers. Scandinavian Journal of Work, Environnement and Health, 24 (3), 134-140.

Prunier-Poulmaire, S., \& Gadbois, C. (2004). Temps et rythmes de travail. In E. Brangier, A. Lancry, \& C. Louche (Eds.), Traité de Psychologie du Travail. Nancy : PUN.

Quéinnec, Y., Teiger, C., \& de Terssac, G. (2008). Repères pour négocier le travail posté (2 ème édition). Toulouse : Octarès.

\section{RESÚMENES}

La previsión de la puesta en marcha de una instalación nuclear de última generación, que está en construcción, suscitó la necesidad de una intervención ergonómica para pensar el diseño de un sistema de trabajo por turnos rotativo $24 \mathrm{~h} / 24$. Se realizó un estudio, en tres plantas existentes, mediante observaciones de las actividades de los diferentes oficios del personal de operación que aseguran el funcionamiento. Los datos colectados muestran diferencias importantes entre dichos oficios en términos de exigencias físicas y mentales de las tareas efectuadas y de su dinámica. Los resultados revelan además una fuerte interdependencia entre las temporalidades, propias a cada uno de los oficios del personal de operación, sujetos al trabajo por turnos rotativos, con los equipos de trabajo de mantenimiento, sujetos ellos a horarios diurnos fijos. También se observan diferencias importantes según los turnos y el estado de funcionamiento del reactor (en paro o no). La gestión de los recursos humanos aparece también como un elemento que se debe considerar en la organización del tiempo del trabajo ya que puede tener un impacto en los desvíos entre los turnos previstos y los turnos realizados. En un enfoque multifactorial y sistémico, el conjunto de dichos resultados constituyen, junto con los conocimientos generales de la cronobiología y de las características propias de la población del personal de operación, uno de los componentes necesarios para la definición de la organización del tiempo de trabajo de la planta que está en construcción.

A previsão do arranque de uma instalação nuclear de última geração, em construção, suscitou a necessidade de uma intervenção ergonómica para pensar a concepção de um sistema de trabalho por turnos rotativos $24 \mathrm{~h} / 24 \mathrm{~h}$. Realizou-se um estudo em três instalações existentes, através de observações das actividades dos diferentes operacionais que asseguram o funcionamento. Os dados recolhidos mostram diferenças importantes nestas actividades, em termos de exigências físicas e mentais das tarefas efectuadas e sua dinâmica. Os resultados revelam também uma forte interdependência entre as temporalidades específicas de cada actividade dos operacionais, sujeitas ao trabalho por turnos rotativos, com as equipas de manutenção, sujeitas a horários diurnos fixos. Também se observam diferenças significativas de acordo com os turnos e o estado 
de funcionamento do reactor (em paragem ou não). A gestão de recursos humanos também aparece como um elemento a ser considerado na organização do tempo de trabalho, já que pode ter um impacto sobre os desvios entre os turnos previstos e os turnos realizados. Numa abordagem multifactorial e sistémica, o conjunto destes resultados constitui, juntamente com o conhecimento geral da cronobiologia e das características próprias da população de operacionais, um dos componentes necessários para a definição da organização do tempo de trabalho da instalação que está em construção.

La prévision de la mise en place d'une installation nucléaire de nouvelle génération, en cours de construction, a suscité le besoin d'une intervention ergonomique pour la conception d'un système d'horaires postés en continu $24 \mathrm{~h} / 24$. Dans ce cadre une étude a été menée dans 3 installations existantes, au moyen d'observations des activités propres aux différents métiers qui en assurent le fonctionnement. Les données ainsi recueillies mettent en lumière de grandes différences entre ces métiers sous l'angle des exigences physiques et mentales des tâches effectuées et de leur dynamique. Elles font en même temps ressortir l'impératif d'une forte interdépendance des temporalités propres à chacun des métiers composant les équipes de conduite et de celles-ci, assujetties à des horaires postés, avec les équipes de maintenance qui suivent des horaires diurnes fixes. De fortes différences sont également enregistrées en fonction des quarts et des étapes de fonctionnement des installations. Les aléas de la gestion des effectifs sont aussi la source d'écarts parfois importants entre le roulement théorique et le roulement effectif. Dans une approche multifactorielle et systémique l'ensemble de ces résultats constitueront avec les connaissances générales de la chronobiologie et les caractéristiques propres de la population des agents concernés une des composantes nécessaires à la définition de l'organisation du temps de travail de l'installation en cours de construction.

The construction of a new generation of nuclear power plant has implied an ergonomics contribution in order to define the shift work schedule for the future operation crew. The study was carried out in three existing nuclear power plants through observation of activities specific to the concerned operating professionals. The data collected highlights major disparities between the performed tasks and their dynamics in terms of physical and mental demands. Furthermore, the same data underlines a strong temporal interdependence amidst the operation crew based on shift work schedules and the maintenance crew organized with set day time schedules. Vast differences are also recorded in terms of physical and mental demands of the tasks performed between day and night shifts as well as between the different stages of nuclear installations. The difficulties of workforce management are also at the source of large differences concerning the theoretical shift work schedule and the shift work schedule in place. In a multifactorial and systemic approach, with the general knowledge of chronobiology and the characteristics of the population involved, the outcomes will be a necessary component to define the shift work schedule for the operation crew of the nuclear power plant in construction.

\section{ÍNDICE}

Palavras-chave: trabalho por turnos, biorritmo, saúde, segurança, central nuclear, concepção, organização do trabalho

Mots-clés: travail posté, travail de nuit, centrale nucléaire, conception, organisation du temps de travail, santé, biorythme

Palabras claves: trabajo por turnos, biorritmo, salud, seguridad, central nuclear, diseño, organización del trabajo

Keywords: shift work, Biorhythm, health and safety, nuclear power plant, design, work organization 


\section{AUTORES}

\section{SOPHIE PRUNIER-POULMAIRE}

UFR de Sciences Psychologiques et de Sciences de l'Education - 200, Av. de la République, 92001 Nanterre Cedex - France.

sophie.prunier-poulmaire@wanadoo.fr

\section{AURORE GHESQUIÈRE}

Université Paris Ouest Nanterre La Défense - 200, Avenue de la République, 92001 Nanterre Cedex - France.

aurore.ghesquiere@gmail.com

\section{CECÍLIA DE LA GARZA}

EDF R\&D - Management des Risques Industriels - 1, Av. Général de Gaulle, 92140 Clamart - France cecilia.de-la-garza@edf.fr

\section{CHARLES GADBOIS}

GRESTO/Conservatoire National des Arts et Métiers, 41 Rue Gay-Lussac, Paris - France gadbois.charles@wanadoo.fr 